\title{
Pour en lire plus : Autoformation et connaissance.
}

Orane Bischoff

\section{(2) OpenEdition \\ Journals}

Édition électronique

URL : https://journals.openedition.org/ere/6184

DOI : $10.4000 /$ ere.6184

ISSN : 2561-2271

Éditeur

Centr'ERE

Référence électronique

Orane Bischoff, «Pour en lire plus : Autoformation et connaissance. », Éducation relative à

l'environnement [En ligne], Volume 16 - 1 | 2021, mis en ligne le 10 mars 2021, consulté le 28 mai 2021.

URL : http://journals.openedition.org/ere/6184; DOI : https://doi.org/10.4000/ere.6184 


\title{
Pour en lire plus : Autoformation et connaissance.
}

\author{
Orane Bischoff
}

\section{RÉFÉRENCE}

Pascal Galvani (2020). Autoformation et connaissance. Lyon : Éditions Chronique Sociale, collection « Pédagogie Formation », 288 pages. [Préface de Gaston Pineau]

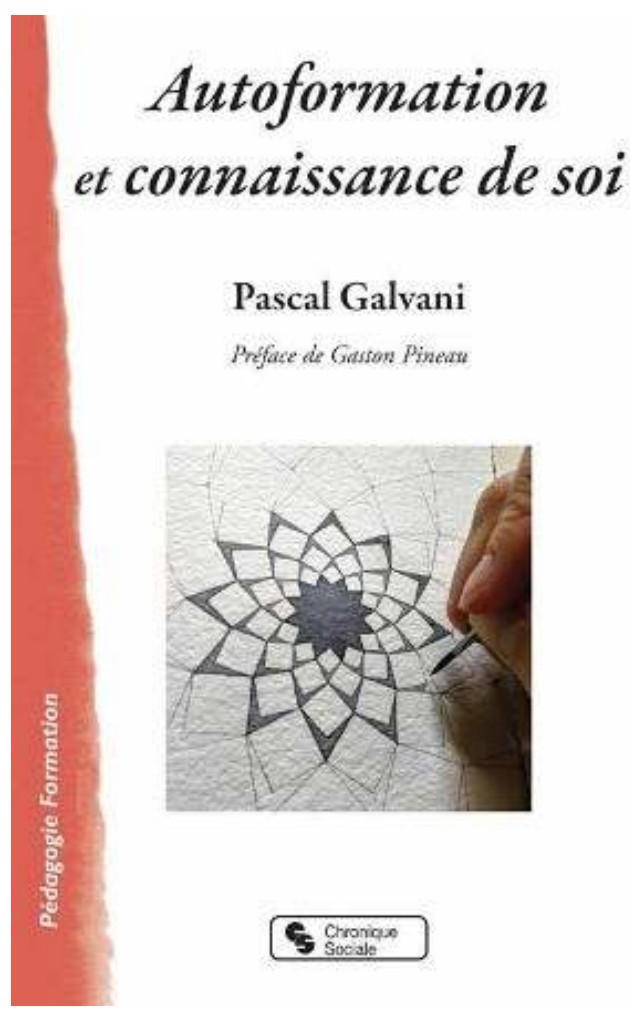


1 Autoformation et connaissance de soi est un ouvrage qui documente finement les concepts et processus en jeu au cœur de la formation existentielle au contact des autres et du monde.

2 S'inscrivant dans la lignée de l'éducation populaire et de figures emblématiques telles que Joffre Dumazedier, Henri Desroche et Bertrand Schwartz, Pascal Galvani ancre son propos au sein des histoires de vie telles que développées par Gaston Pineau et la recherche-action existentielle de René Barbier. Il prend appui sur des méthodologies «maïeuticiennes » qui ont fait leurs preuves dans des formations pour adulte, celles qui avaient pour objectif de mettre l'étudiant en position de chercheur sur son propre parcours, sa propre trajectoire afin d'en faire le creuset d'une exploration méthodique sur la base d'une question propre formulée en problématique. Le travail est nourri par le dialogue entre étudiants co-chercheurs, le chercheur professionnel (formant une coopérative de productions de savoirs) et des éléments théoriques.

3 Au travers de son parcours d'enseignant-chercheur, Pascal Galvani nous offre une synthèse de ses travaux qui lui ont permis d'aboutir aux perspectives méthodologiques qu'il nous présente et qu'il a pu explorer auprès de nombreux groupes. C'est donc un ouvrage ancré dans la pratique richement étayé de références théoriques.

4 L'auteur pose son cadre épistémologique, élément fondamental pour comprendre sa démarche. Pour plonger au coeur de l'expérience formatrice de la personne et comprendre ce qui se joue, Pascal Galvani mobilise la phénoménologie herméneutique. Les processus en jeu semblent infiniment riches et il faut alors avoir un regard systémique ancré dans l'approche complexe telle que définie par Morin.

5 L'auteur nous invite ensuite à mieux appréhender la dimension biocognitive de cette formation au sein d'une dynamique tripolaire déclinée en auto-socio-éco formation abordée par les trois niveaux de réalité: cognitif, sensori-moteur et imaginaire/ poétique.

6 Tout au long de l'ouvrage, l'auteur précise sa définition de l'autoformation de sorte que celle-ci s'enrichit au fil des chapitres. Il nous présente l'autoformation comme « une dynamique de prise de conscience émancipatrice » et comme «la prise de conscience émancipatrice des habitus et des schèmes d'interaction que chacun développe avec le monde physique et social ».

7 Pascal Galvani nous accompagne pas à pas dans ces processus autoformateurs. D'abord en investissant la question de l'autos, et plus précisément la question du soi. L'autoformation est envisagée comme un trajet anthropologique qui met en dialogue le $\mathrm{je} / \mathrm{moi} / \mathrm{soi}, \mathrm{l}^{\prime i p s e ́ ~ e t ~ l ' i d e m ~}{ }^{1}$ de la personne. Pour l'auteur, ce qui se joue dépasse le je et le moi et vient construire un soi plus large plus englobant, qui intègre les autres et le monde. Mais pour s'inscrire réellement dans l'autoformation, cela nécessite un retour réflexif sur soi afin de saisir les instants créateurs, décisifs où la conscience de soi semble élargie tout en étant présente et investie. Pascal Galvani nous présente ces méthodes d'exploration des expériences autoformatrices comme notamment l'atelier des Kaïros traité sous forme individuelle et collective, impliquant des mises en dialogue et résonances entre participants grâce à des cercles de parole aux consignes précises. Les kaïros sont présentés comme étant des «instants décisifs où tout se joue au coeur de nos expériences formatrices intenses ». Des techniques sont présentées, comme les «je me souviens» qui facilitent la quête de souvenirs, l'émergence de moments signifiants où s'est manifestée cette « intelligence de l'agir ». En mobilisant la méthode 
de l'entretien d'explicitation de Vermersch, l'auteur nous montre comment nous pouvons ensuite "remonter" au coeur de l'expérience phénoménologique pour en saisir la richesse et permettre une conscientisation profonde de ce qui s'est joué au coeur de l'action.

Il s'agit ensuite d'accompagner la mise en "sens » de ces moments explicités par une mise en résonance et en dialogue entre participants, sur les plans «pratique, émotionnel, symbolique et cognitif ".

9 Pour l'auteur, dans cette démarche globale de recherche-action-formation existentielle, l'enjeu est l'émancipation par conscientisation des conditions socio-historiques afin de pouvoir s'ouvrir à l'intercompréhension au sein d'un groupe, d'une communauté. À l'heure de la mondialisation et d'une crise planétaire sociale et écologique, il semble nécessaire d'offrir des espaces de dialogue, de «croisement réciproque des modes de subjectivation" pour nourrir un soi terrien, humain, qui permettrait une " métamorphose des consciences ».

10 Grâce à cet ouvrage, les chercheurs et praticiens de l'Éducation relative à l'environnement qui s'intéressent à l'écoformation, vont trouver des cadres, outils et méthodes pour explorer de façon rigoureuse nos relations et expériences terrestres. Nous avons le fil d'Ariane, le scaphandrier et le protocole pour explorer «comment nous sommes affectés, touchés, formés, éduqués par la Terre ». Il ne reste plus qu’à pratiquer et s'entraîner!

\section{NOTES}

1. Idem : l'identité qui s'inscrit dans la continuité. Ipsée : la singularité de soi à chaque instant.

\section{AUTEUR}

\section{ORANE BISCHOFF}

Formatrice, ingénieure d'étude pédagogique, Orane Bischoff est doctorante en sciences de l'éducation à l'institut Agro, Montpellier SupAgro, campus de Florac 